REVISTA INTERNACIONAL DE CIENCIAS DEL DEPORTE International Journal of Sport Science

doi:10.5232/ricyde2007.00704

Rev. int. cienc. deporte

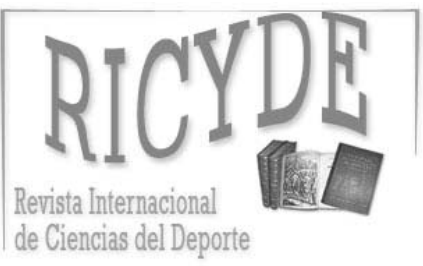

International Journal of Sport Science VOLUMEN III. AÑO III

Páginas:34-48 ISSN:1885-3137 No 7 - Abril - 2007

\title{
Validación de una escala reducida de utilidad percibida de la práctica de la actividad física y el deporte. \\ Validation of a reduce scale of perceived utility of physical activity and sport practice.
}

\author{
Arribas Galarraga, Silvia \\ Arruza Gabilondo, José Antonio \\ González Rodriguez, Oscar \\ Telletxea Arzamendi, Saioa \\ Universidad del Pais Vasco \\ (U.P.V./E.H.U.)
}

\section{Resumen}

En este estudio se presenta la validación de una versión reducida a partir de la Escala de Utilidad Percibida de la Actividad Física y el Deporte desarrollado por Sánchez, Mendizabal y Velasco en el año 2000. Participaron en este estudio 3.080 jóvenes de ambos sexos de entre quince y dieciocho años que cursaban sus estudios en Gipuzkoa. El análisis exploratorio realizado permitió la obtención de 4 grandes dimensiones: Socialización, Sensación de Bienestar, Forma física-salud y Liberación de Tensiones. Este modelo se ratifico mediante un análisis confirmatorio quedando reducida la escala a 12 ítems y 4 dimensiones. Estas dimensiones mostraron relaciones diversas con otras dimensiones motivacionales que forman parte de otros instrumentos como el cuestionario CDPD (Duda y Nicholls, 1992) o la escala CSRD (Treasure y Roberts, 1994). Este estudio ha consolidado el interés de este instrumento y su utilidad para analizar las percepciones de los ciudadanos sobre el papel que las actividades físicas pueden tener en su salud y sus vidas.

\section{Abstract}

The main purpose of this study was to validate a short form of a Perceived utility of Physical Activity and Sport Scale with the reference of the Sanchez, Mendizabal and Velasco's scale (2000). Three thousand and eighty younger people participated in this study ( 15 to 18 years old) from Gipuzkoa (Vasc Country). Exploratory analysis (EFA) permitted to get a factorial solution of four factors: Socialization, Wellness, health and Fitness and Tension reduction. This model was demonstrated by a Confirmatory factor analysis (CFA) with an scale of twelve items and four dimensions. These dimensions manifested different relationships with other motivational dimensions like the Duda \& Nicholls' CDPD questionnaire (1992) and the Treasure \& Roberts' CSRD scale (1994). This study has consolidated the interest of this tool and its utility as an instrument for knowing the perceptions of citizens about the role of physical activity and sport to their health and life.

Key words:perceived utility, physical activity and sport, scale and assessment, motivation to sport.

Palabras clave: utilidad percibida, práctica deportiva, actividad física, escala de medición, motivación hacia el deporte.

Correspondencia/correspondence: José Antonio Arruza Gabilondo.

Escuela Universitaria de Magisterio de Donostia.

E-mail: jarruza@ehu.es 


\section{Introducción}

$\mathrm{H}$ oy en día, y como ocurre en la mayoría de las culturas occidentales en las que el nivel socioeconómico es medio-alto, se ha producido una involución de lo físico a favor de lo tecnológico, hecho, que se ha visto reflejado en el creciente sedentarismo de nuestras sociedades. Como consecuencia de ello, surgen nuevas tendencias que nos hablan sobre el bienestar y la calidad de vida, y en la mayoría de los discursos, ambos aspectos se unen a la práctica de la actividad física y el deporte (en adelante PAFYD), lo que hace despertar el interés y la necesidad de abordar la percepción sobre la utilidad de la práctica deportiva con una perspectiva científica. En este sentido, hemos considerado adecuado partir del trabajo que Sánchez, Mendizábal y Velasco (2000) realizaron con su "escala para la medición de la utilidad percibida de la práctica del deporte", asociada al tipo de práctica que se realiza.

Con la ayuda de 10 expertos del ámbito de la actividad física y el deporte, configuraron una escala constituida por 40 ítems agrupados en 8 factores, que cuantifican la percepción que se tiene sobre la utilidad de la PAFYD, y son: Liberación e identificación, Desarrollo personal, Búsqueda de experiencias, Forma física y salud, Socialización y relación, Evasión, Riesgo, Competición

Primeramente realizamos un estudio en el año 2001, sobre una muestra de 400 sujetos residentes en Gipuzkoa. La selección de dicha muestra se hizo en base a los datos del Instituto Vasco de Estadística (EUSTAT), con un muestreo aleatorio estratificado, de la población mayor de 15 años. Se utilizó un diseño descriptivo y valorativo con metodología transversal, con un nivel de error muestral correspondiente a un nivel de confianza del 95,5\%.

Los datos que obtuvimos fueron comparados con los presentados por Sánchez Bañuelos, Mendizabal y Velasco (2000), con un resultado favorable. Como consecuencia de ello, nos planteamos la posibilidad de diseñar una versión abreviada de la escala original para la medición de la "Utilidad percibida de la práctica de la actividad física y el deporte" que, manteniendo la validez y fiabilidad mostrada, tanto por los autores de la escala (Sánchez, Mendizábal y Velasco, 2000) como por el trabajo realizado por Arruza y Arribas (2001) señalado, nos facilitara información relevante acerca de las creencias sobre la utilidad de la PAFYD en nuestro entorno.

Nos hemos basado principalmente en la Teoría de Auto-Eficacia (Bandura 1982), la Teoría de la Acción Razonada (Fishbein y Ajzen 1975) y la Teoría de la Acción Planificada (Ajzen y Madeen 1986; Ajzen 1985,1987,1988) ya que consideramos que las creencias de eficacia, influyen sobre el modo de pensar, sentir, motivarse y actuar de las personas. Estas creencias tendrán influencia sobre la elección de conductas, el esfuerzo que se emplee en la realización material de las conductas elegidas, e incluso en la persistencia ante las diversas dificultades que pudieran presentarse. También en los planteamientos que tienen que ver con la toma de decisiones, aparecen estas dos últimas teorías. 
Arribas, S.; Arruza, J.A.; González, O.; Telletxea, S. Validación de una escala reducida de utilidad percibida de la práctica de la actividad física y el deporte. Revista Internacional de Ciencias del Deporte. 7(3), 34-48 http://www.cafyd.com/REVISTA/00704.pdf

La teoría de la acción razonada, se encuentra subordinada a la voluntad individual y nos ayudará a entender el comportamiento. En la teoría de la acción planificada, existen ciertos conflictoscontrol que se encuentran fuera de la propia voluntad del individuo y que también serán útiles para explicar el comportamiento de los sujetos. Los modelos que más se han desarrollado y a los que nosotros prestaremos mayor atención son, los modelos sobre Utilidad y Valor Esperado (Kuhl 1982; Abelson y Levi 1985; Eiser 1988). En este sentido Eagly y Chaikens (1993) manifiestan que los sujetos presentan actitudes positivas hacia aquellas cosas, personas, situaciones o conductas que creen que tienen atributos positivos.

En el ámbito de la PAFYD tales factores están relacionados entre otros con la diversión y la satisfacción que pueden influir sobre las creencias de utilidad, lo que nos lleva a establecer una intención que derivará en un determinado comportamiento que en este contexto se puede materializar a través de la realización de la práctica, cambiar el tipo de práctica o incluso abandonar dicha práctica. Por tanto, consideramos como se indica en la figura siguiente (figura 1), que el valor por expectativa o valor esperado, al igual que la autoeficacia, influyen en las intenciones y actitudes, ya que estas inciden de forma relevante en el comportamiento de los sujetos y en las creencias que los sujetos tienen sobre la utilidad de la PAFYD, que a nuestro entender están estrechamente unidas a la satisfacción y a la diversión que los practicantes experimentan.

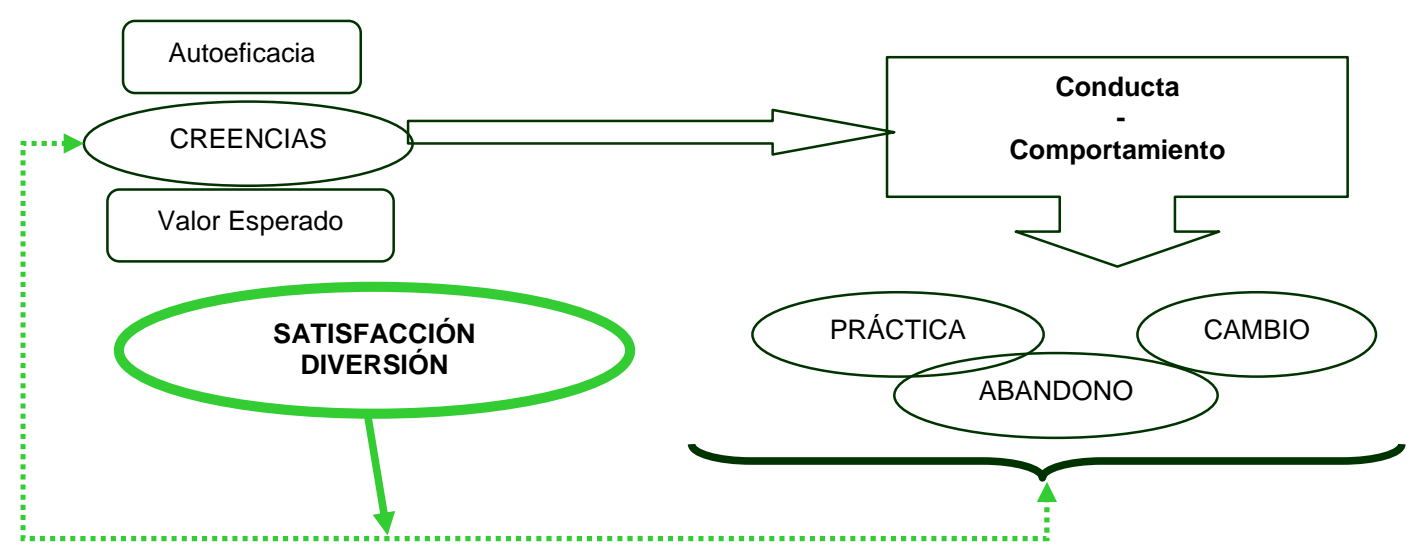

Figura 1. Relación de las teorías de Acción Razonada, Acción Planificada, y Auto-Eficacia con las creencias de utilidad de la PAFYD 


\section{Metodología}

\section{Muestra}

La muestra de este estudio recogida durante el año 2002 está compuesta por 3.080 jóvenes de ambos sexos de entre quince dieciocho años que cursaban sus estudios en Gipuzkoa (País Vasco). El universo de esta muestra lo componen un total de 24.920 sujetos. Para determinar el tamaño de la muestra se tomo el margen de error muestral para el total de la muestra de $+/-3 \%$, para a un margen de confianza del 95.5\% calculado según las tablas de Arkin y Colton (1962) y bajo el supuesto de máxima indeterminación $(\mathrm{p}=\mathrm{q}=50 \%)$. El método de muestreo utilizado fue el muestreo aleatorio estratificado de afijación proporcional.

\section{Variables}

Las variables seleccionadas han sido: Las creencias de utilidad de la PAFYD, la Diversión, la Satisfacción.

\section{Instrumentos}

Se ha elaborado un cuestionario ad hoc con los instrumentos seleccionados. Para medir las creencias de utilidad de la PAFYD, se ha elegido el cuestionario reducido de utilidad percibida de la PAFYD (en adelante CRUP/PAFYD en una escala porcentual de 0 (totalmente en desacuerdo) a 100 (totalmente de acuerdo). El grado de diversión que generaba la PAFYD en los practicantes se registró a través del cuestionario CDPD (Duda y Nicholls, 1992) en una escala porcentual de 0 (totalmente en desacuerdo) a 100 (totalmente de acuerdo). El nivel de satisfacción se obtuvo a través de la escala CSRD (Treasure y Roberts, 1994) en una escala de 0 (Nada) a 100 (Mucho).

\section{Procedimiento}

Se realizó un primer contacto con los centros escolares que impartieran clase a los chicos y chicas guipuzcoanos de edades comprendidas entre los 15-18 años. Una vez recibida la conformidad del centro y establecida la cita, nos desplazábamos para realizar la pasación del cuestionario y la toma de datos.

\section{Análisis estadísticos}

Los análisis estadísticos realizados con el fin de establecer la fiabilidad y validez de la escala CRUP/PAFYD, se pueden dividir en 4 secciones: 
Arribas, S.; Arruza, J.A.; González, O.; Telletxea, S. Validación de una escala reducida de utilidad percibida de la práctica de la actividad física y el deporte. Revista Internacional de Ciencias del Deporte. 7(3), 34-48 http://www.cafyd.com/REVISTA/00704.pdf

Primeramente, se llevó a cabo un análisis de componentes principales. Es una técnica que permite transformar un conjunto de variables intercorrelacionadas en otro conjunto de variables (factores) para reducir la información disponible sobre un conjunto de individuos en los cuales se han tomado diversas observaciones.

A continuación se llevó a cabo un análisis de consistencia interna para determinar el coeficiente alfa de cada dimensión por una parte y del total de la escala por otra.

En tercer lugar, para fortalecer la validez del cuestionario y confirmar su estructura factorial (Vallerand y Richer, 1987), se realizó un análisis factorial confirmatorio por medio del programa AMOS 5.0.

En cuarto y último lugar se llevó a cabo un análisis correlacional. En él se correlacionaron las distintas dimensiones de la escala CRUP/PAFYD con las dimensiones de diversión y satisfacción analizadas con el fin de establecer las posibles relaciones entre todas ellas.

\section{Resultados}

\section{Análisis Componentes Principales}

Para contrastar de forma empírica nuestra hipótesis respecto a la estructura interna del instrumento, se llevó a cabo un análisis factorial de componentes principales. Se obtuvieron unos índices de adecuación muestral KMO (Kaiser Meyer Olkin) de 0,92 (Tabla 1), y según Martínez Arias (1996) un índice por encima de 0,85 determina que dicha medida tiene un nivel muy bueno para continuar con el análisis de componentes principales

\begin{tabular}{|c|c|c|}
\hline $\begin{array}{l}\text { Medida de adecuación muestral de Kaiser- } \\
\text { Meyer-Olkin }\end{array}$ & & .92 \\
\hline $\begin{array}{l}\text { Prueba de esfericidad } \\
\text { de Bartlett }\end{array}$ & $\begin{array}{l}\text { Chi-cuadrado } \\
\text { aproximado }\end{array}$ & 18202,12 \\
\hline & gl & 78 \\
\hline & Sig. & .000 \\
\hline
\end{tabular}

Tabla 1. Medida de adecuación muestral de KMO y prueba de esfericidad de Bartlett.

Posteriormente, se llevó a cabo un análisis de componentes principales con rotación Varimax, obteniéndose 4 factores que en total explican una varianza de $71.18 \%$. La estructura de agrupamiento de los ítems dentro de los 4 factores hallados, se puede encontrar en la matriz de componentes rotados (Tabla 2). Se han omitido los coeficientes menores a 0.36 para una mejor visualización de los ítems que presentan mayor saturación en cada uno de los factores. Según se observa en la tabla 2, los 12 ítems parecen tener un alto peso factorial en el factor o dimensión 
Arribas, S.; Arruza, J.A.; González, O.; Telletxea, S. Validación de una escala reducida de utilidad percibida de la práctica de la actividad física y el deporte. Revista Internacional de Ciencias del Deporte. 7(3), 34-48 http://www.cafyd.com/REVISTA/00704.pdf

que representa o trata de representar. Pero también se observa que el ítem "divertirse" posee un peso factorial bajo. Posiblemente el carácter instrumental que sugiere el concepto "utilidad" pueda explicar el bajo peso que presenta este ítem.

\begin{tabular}{|l|c|c|c|c|}
\hline & \multicolumn{3}{|c|}{ Componentes } \\
\hline & $\mathbf{1}$ & $\mathbf{2}$ & $\mathbf{3}$ & $\mathbf{4}$ \\
\hline Olvidar las preocupaciones & & & & .81 \\
\hline $\begin{array}{l}\text { Desarrollar un mejor concepto de } \\
\text { uno mismo. }\end{array}$ & & .67 & & .41 \\
\hline Mejorar o mantener la forma física. & & & .87 & \\
\hline $\begin{array}{l}\text { Lograr una mayor seguridad en uno } \\
\text { mismo. }\end{array}$ & & .74 & & \\
\hline Desarrollar el afán de superación. & & .73 & & \\
\hline Comunicarse con los demás. & .77 & & & \\
\hline Ayudar mejor a los demás. & .75 & .38 & & \\
\hline Desahogarse. & .41 & & & .71 \\
\hline Dar salida al afán competitivo & .44 & .44 & & \\
\hline Hacer nuevos amigos. & .81 & & & \\
\hline Expresarse con libertad. & .66 & & & \\
\hline Mejorar y mantener la buena salud. & & & .84 & \\
\hline
\end{tabular}

Tabla 2. Matriz de componentes rotados.

\section{Análisis de fiabilidad}

Para analizar la fiabilidad del cuestionario, se utilizó el coeficiente alpha de Cronbach (1951), considerado el más adecuado para instrumentos cuya puntuación final se obtiene mediante el proceso sumativo o de acumulación de puntos Nunnally (1976).

\begin{tabular}{|l|r|r|}
\hline Escala & Hombres & Mujeres \\
\hline CRUP/PAFYD (Arribas, Arruza) & & \\
\hline Socialización & .82 & .89 \\
\hline Sensación de bienestar & .78 & .85 \\
\hline Forma física y salud & .81 & .83 \\
\hline Liberación de tensiones & .50 & .73 \\
\hline Total & $\mathbf{. 8 9}$ &. $\mathbf{9 2}$ \\
\hline Cuestionario Utilidad (Bañuelos et al, 2000) & .94 & .95 \\
\hline
\end{tabular}

Tabla 3. Índices de consistencia interna del CRUP/PAFYD en función del sexo. 
Arribas, S.; Arruza, J.A.; González, O.; Telletxea, S. Validación de una escala reducida de utilidad percibida de la práctica de la actividad física y el deporte. Revista Internacional de Ciencias del Deporte. 7(3), 34-48 http://www.cafyd.com/REVISTA/00704.pdf

Como se puede observar en la tabla 3 , se puede apreciar que todos los índices de consistencia interna de las diferentes subescalas se encuentran por encima de 0,7 a excepción del factor "liberación de tensiones" que presenta un índice de 0,5. Teniendo en cuenta el rango establecido por Nunnally (1976) para aceptar la consistencia interna de una escala podemos afirmar que las cuatro dimensiones que componen la CRUP/PAFYD (tabla 3) presentan una consistencia aceptable (a excepción de la ya mencionada) tanto en hombres como mujeres, en las subescalas por separado y en la escala en su conjunto.

Consideramos que existe una correspondencia directa entre la categorización de Sánchez (1987), que consiste en un estudio teórico, con los factores que aparecen en el test de 40 ítems (Sánchez et al., 2000), resultado de un estudio empírico, y los que nosotros proponemos para la escala reducida (CRUP/PAFYD), resultante de los análisis previamente expuestos. Recogemos en la figura 2, a modo de esquema las 5 categorías que se establecen en el primer estudio teórico de Sánchez (1987), los 8 factores que se obtuvieron en el segundo estudio empírico (cuestionario de 40 items), y los 4 factores que hemos obtenido en el presente estudio para la reducción del cuestionario sobre la utilidad percibida de la práctica del deporte a 12 ítems.

\begin{tabular}{|l|l|l|}
\hline \multicolumn{1}{|c|}{ SÁNCHEZ, (1987) } & \multicolumn{1}{|c|}{$\begin{array}{c}\text { SÁNCHEZ, MENDIZÁBAL, } \\
\text { VELASCO (2000) }\end{array}$} & \multicolumn{1}{c|}{ (CRUP/PAFYD) } \\
\hline $\begin{array}{l}\text { 1. Socialización-Relación } \\
\text { con otras personas }\end{array}$ & $\begin{array}{l}\text { 3.Búsqueda de experiencias } \\
\text { 5.Socialización y relación }\end{array}$ & 1.Socialización \\
\hline $\begin{array}{l}\text { 2. Hedonismo-Sensación } \\
\text { de bienestar } \\
\begin{array}{l}\text { 3. Realización personal- } \\
\text { Consecución de metas }\end{array}\end{array}$ & $\begin{array}{l}\text { 2.Desarrollo personal } \\
\text { 8.Competición } \\
\text { 7.Riesgo }\end{array}$ & 2.Sensación de bienestar \\
\hline $\begin{array}{l}\text { 4. Acondicionamiento } \\
\text { físico-salud }\end{array}$ & 4.Forma física y salud & 3. Forma física y salud \\
\hline $\begin{array}{l}\text { 5. Evasión-Liberación de } \\
\text { tensiones }\end{array}$ & $\begin{array}{l}\text { 1.Liberación e identificación } \\
\text { 6. Evasión }\end{array}$ & 4. Liberación de tensiones \\
\hline
\end{tabular}

Figura 2. Denominación de las categorías obtenidas en cada uno de los estudios realizados relacionadas con la utilidad percibida de la PAFYD. 
Arribas, S.; Arruza, J.A.; González, O.; Telletxea, S. Validación de una escala reducida de utilidad percibida de la práctica de la actividad física y el deporte. Revista Internacional de Ciencias del Deporte. 7(3), 34-48 http://www.cafyd.com/REVISTA/00704.pdf

\section{Análisis Factorial Confirmatorio}

Con el fin de establecer los pesos factoriales de los ítems y "confirmar" la estructura factorial o modelo hipotetizado de 4 factores, nos ha parecido importante realizar un análisis factorial confirmatorio. Esto nos ha permitido conocer la magnitud del ajuste de la estructura teorizada a los datos. Para llevar a cado el análisis confirmatorio, se seleccionaron 200 sujetos de la muestra original de 3.080, para evitar por una parte un sobre ajuste de los datos y por otro lado poder obtener un tamaño muestral crítico (Gondar, 2002). Los parámetros del modelo original fueron estimados siguiendo el criterio de máxima verosimilitud.

El modelo propuesto para el cuestionario de la práctica deportiva estaba formado por cuatro variables latentes independientes (Socialización, Sensación de bienestar, Forma física-salud, Liberación de tensiones), y 12 variables independientes observadas correspondientes a los 12 items del cuestionario (ver Anexo 1). En el modelo, cada una de las variables latentes independientes (factores), viene definido por los distintos indicadores observables (items). Las razones que explican esta configuración del modelo, se explican sobre la base de los resultados del análisis de componentes principales. Como se aprecia en la figura 3, las correlaciones cuadráticas son elevadas en todos los items. 
Arribas, S.; Arruza, J.A.; González, O.; Telletxea, S. Validación de una escala reducida de utilidad percibida de la práctica de la actividad física y el deporte. Revista Internacional de Ciencias del Deporte. 7(3), 34-48 http://www.cafyd.com/REVISTA/00704.pdf

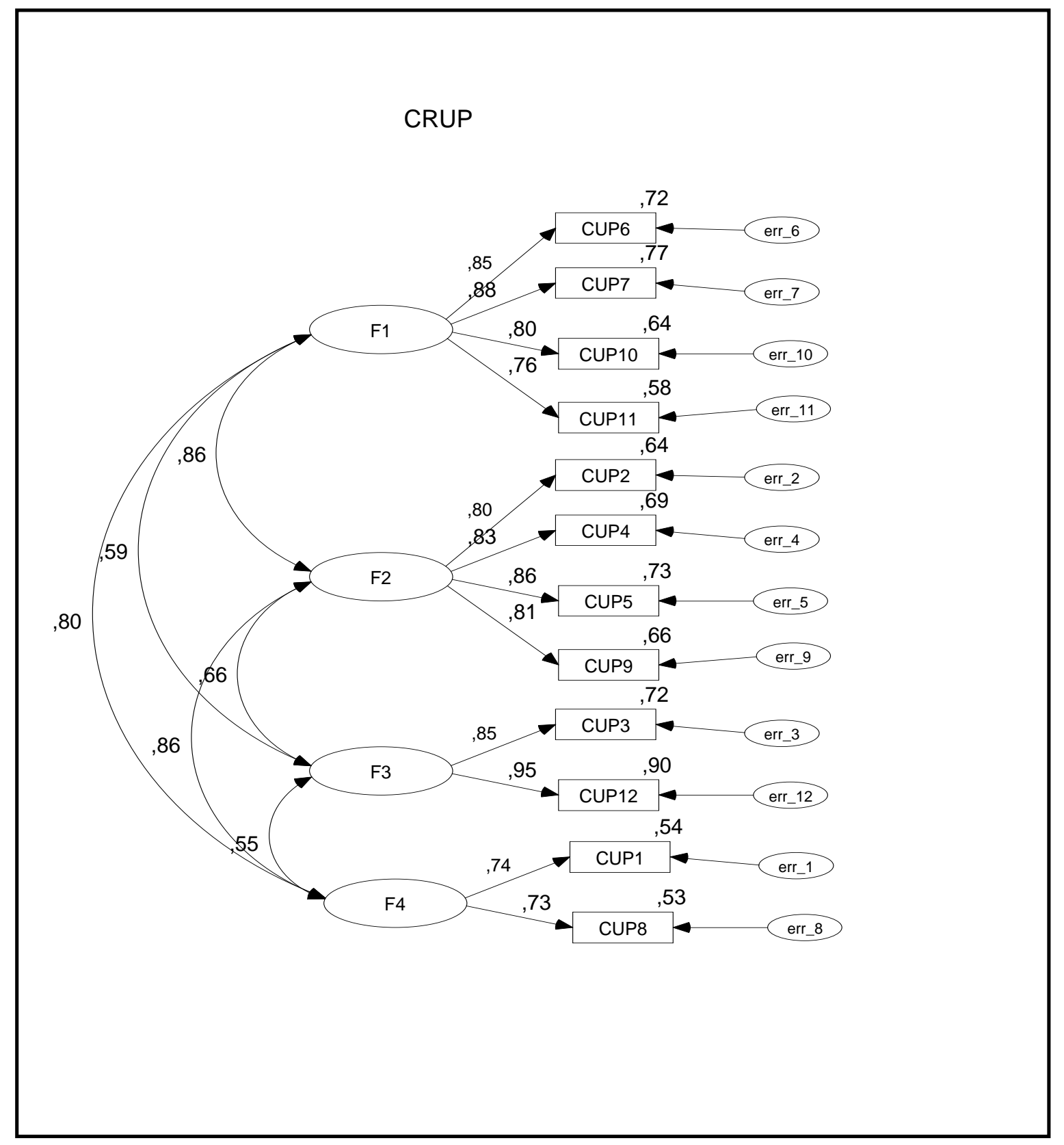

Figura 3. Parámetros estimados para el CRUP.

En la tabla 4, se recoge la información proporcionada, por algunos de los índices de ajuste más utilizados (García Cueto, Gallo y Miranda, 1998; García, Ruiz y Abab, 2003; Sandín y otros, 2003) correspondientes al análisis de componentes reflejados en la figura 1. 
Arribas, S.; Arruza, J.A.; González, O.; Telletxea, S. Validación de una escala reducida de utilidad percibida de la práctica de la actividad física y el deporte. Revista Internacional de Ciencias del Deporte. 7(3), 34-48 http://www.cafyd.com/REVISTA/00704.pdf

\begin{tabular}{|l|c|c|l|l|l|l|}
\hline & $\chi^{2}$ & $\mathbf{P}$ & $\chi \mathbf{2} / \mathbf{g l}$ & RMSEA & NFI & CFI \\
\hline Modelo original & 102.38 & $<0.0001$ & 2.13 & 0.07 & .93 & .96 \\
\hline
\end{tabular}

Tabla 4. Valores correspondientes a los índices del análisis factorial confirmatorio realizado al modelo original y al reespecificado.

Además del estadístico $\chi 2$ (Chi cuadrado) se han considerado de manera complementaria otros índices, como el $\chi 2$ /gl (Chi cuadrado Normalizado), RMSEA (Residual de la raíz cuadrática media de aproximación), NFI (Índice de ajuste normalizado) y CFI (Índice de ajuste comparativo). En el caso de $\chi^{2}$ /gl se consideran aceptables valores por debajo de 4 ; en el caso de NFI y CFI, por encima de 0,90; y, en el caso de del RMSEA por debajo de 0,05. De esta forma se puede comprobar que tanto el $\chi 2 / \mathrm{gl}$ como los índices NFI, CFI presentan valores correctos que permiten determinar una aceptable bondad de ajuste del modelo original.

\section{Análisis correlacional.}

En este punto, comentaremos los resultados obtenidos del análisis correlacional (Correlación de Pearson) de la utilidad percibida de la PAFYD con la diversión y la satisfacción, que se presentan el la tabla 5.

\begin{tabular}{|c|c|c|c|c|}
\hline & \multicolumn{4}{|c|}{ Creencias de utilidad de la PAFYD } \\
\hline & Socialización & $\begin{array}{l}\text { Sensación de } \\
\text { bienestar }\end{array}$ & Forma física-salud & $\begin{array}{c}\text { Liberación de } \\
\text { tensiones }\end{array}$ \\
\hline Diversión & $.58 * *$ & $.59 * *$ & $42 * *$ & $.53 * *$ \\
\hline Satisfacción & & & & \\
\hline $\begin{array}{l}\text { Experiencias } \\
\text { de maestría }\end{array}$ & $.58 * *$ & $.66 * *$ & $.50 * *$ & $.56 * *$ \\
\hline $\begin{array}{l}\text { Aprobación } \\
\text { social }\end{array}$ & $.48 * *$ & $.53 * *$ & $.42 * *$ & $46 * *$ \\
\hline $\begin{array}{l}\text { Éxito } \\
\text { normativo }\end{array}$ & $.40 * *$ & $.48^{* *}$ & $.37 * *$ & $40 * *$ \\
\hline
\end{tabular}

Tabla 5. Valores del grado de correlación y del nivel de significación entre las dimensiones de la PAFYD, con las variables Diversión y Satisfacción.

** $(\mathrm{p} \leq .01)$

$* * *(\mathrm{p} \leq .001)$ 
Arribas, S.; Arruza, J.A.; González, O.; Telletxea, S. Validación de una escala reducida de utilidad percibida de la práctica de la actividad física y el deporte. Revista Internacional de Ciencias del Deporte. 7(3), 34-48 http://www.cafyd.com/REVISTA/00704.pdf

Centrándonos en el valor de la correlación de las dimensiones correspondientes a la utilidad percibida, cabe resaltar la intensidad alta que se da entre la sensación de bienestar-consecución de metas $\left(\mathrm{r}=0.59^{* *}\right)$, la socialización-relación $\left(\mathrm{r}=0.58^{* *}\right)$, y moderadamente alta que se da con la evasión-liberación de tensiones $\left(\mathrm{r}=0.43^{* *}\right)$ y la forma física-salud $\left(0.42^{* *}\right)$, con la diversión.

Los datos obtenidos entre las dimensiones de satisfacción con las de utilidad percibida, muestran una correlación estadísticamente significativa entre todas ellas. Destacaremos los altos índices en cuanto a la intensidad en la correlación entre la sensación de bienestar $\left(\mathrm{r}=0.66^{* *}\right)$, la liberación de tensiones $\left(\mathrm{r}=0.56^{* *}\right)$, la socialización $\left(\mathrm{r}=0.58^{* *}\right)$, y la forma física-salud $\left(0.50^{* *}\right)$ con las experiencias de maestría.

En el caso de la aprobación social los valores de la correlación son ligeramente superiores a la dimensión anterior como podemos observar en la tabla 5, con la sensación de bienestar $\left(\mathrm{r}=0.53^{* *}\right)$, con la socialización $\left(\mathrm{r}=0.48^{* *}\right)$ con la liberación de tensiones $\left(\mathrm{r}=0.46^{* *}\right)$, y con la forma física-salud $(0.42 * *)$.

En cuanto al éxito normativo vemos que el valor de la correlación es el más bajo, aunque de intensidad moderadamente alta, concretamente, con la sensación de bienestar $\left(\mathrm{r}=0.48^{* *}\right), \mathrm{y}$ moderada con la socialización $\left(\mathrm{r}=0.40^{* *}\right)$, con la liberación de tensiones $\left(\mathrm{r}=0.40^{* *}\right)$ y la forma física-salud $\left(0.37^{* *}\right)$.

\section{Discusión}

El interés principal de la presente investigación se ha centrado en configurar una escala abreviada que mida las creencias de utilidad acerca de la práctica de la actividad física y el deporte, a partir de los estudios realizados por Sánchez (1987) y Sánchez, Mendizábal y Velasco (2000). Los resultados alcanzados, nos permiten plantear una escala reducida de utilidad de la práctica deportiva con 4 factores: "Socialización", "Sensación de bienestar", "Forma físicaSalud", y "Liberación de tensiones", con una alta fiabilidad y validez. Además, en el análisis factorial de componentes principales también se han mantenido 4 dimensiones que se corresponden con dicho modelo teórico. Se ha conseguido una reducción del $70 \%$ en la longitud del cuestionario dándose una pérdida centesimal en el coeficiente alfa de Crombach $(0,04$ en el caso de los hombres y 0,03 en el caso de las mujeres) lo que consolida la fiabilidad del cuestionario. El empleo del análisis factorial confirmatorio, ha permitido comprobar y reforzar la configuración de la escala de acuerdo al modelo teórico propuesto inicialmente, apoyado en la opinión de diversos expertos, en los resultados de los estudios previos realizados, así como en la bibliografía consultada.

Esto nos permite afirmar que la escala reducida finalmente propuesta, podrá ser aplicada de forma ágil y eficiente, al estar configurada por tan sólo 12 items, frente a la originaria de 40 items. Es cierto que todavía se puede mejorar algunos índices psicométricos con nuevos estudios, 
Arribas, S.; Arruza, J.A.; González, O.; Telletxea, S. Validación de una escala reducida de utilidad percibida de la práctica de la actividad física y el deporte. Revista Internacional de Ciencias del Deporte. 7(3), 34-48 http://www.cafyd.com/REVISTA/00704.pdf

pero consideramos que los obtenidos en esta investigación garantizan una alta fiabilidad a la hora de valorar la utilidad percibida de la práctica de la actividad física y el deporte en el ámbito de una población concreta.

También es muy interesante la correlación que se ha observado con las variables diversión y satisfacción, lo que ayuda a comprender con mayor profundidad los motivos por los cuales las personas se orientan hacia dicha práctica. Se ha partido de un modelo teórico habitual relacionado con la práctica deportiva, lo que permite avanzar y reforzar el conocimiento existente entre las creencias personales asociadas a variables motivacionales relacionadas con la práctica de la actividad física y el deporte.

Es positivo que la correlación entre el factor Sensación de bienestar con las tres dimensiones de la satisfacción, y con la diversión, presenten siempre mayores valores que el resto de los factores, lo que refuerza a pesar de su reducción, tanto la validez externa de la escala, como la congruencia interna.

También hemos observado que presenta un orden lógico en cuanto a la intensidad de los valores de la correlación entre los factores de utilidad percibida con las variables satisfacción y diversión, es decir, los valores del factor Socialización se sitúan en segundo lugar, los de Liberación de tensiones en tercer lugar y por último, en cuarto lugar, los correspondientes a Forma físicaSalud. Se percibe una lógica interna a la hora de aplicar este instrumento ya que los valores del grado de correlación son positivos y significativos, lo que supone que a medida que aumenta la satisfacción y la diversión por la práctica deportiva se incrementa el valor de las creencias sobre su utilidad.

Pensamos que se debe continuar con esta línea de investigación, desarrollando nuevos proyectos en los que se utilice este nuevo instrumento y nos ayude a detectar las fluctuaciones que se puedan producir en dichas creencias.

\section{Bibliografia}

Abelson, R.P., y Levi I. A. (1985). Making a Decision Theory. En G. Lindzey y E. Aronson (Eds). Handbook of Social psychology. Vol 1. New York: Addison Wesley.

Ajzen, I. (1985). From intentions to actions: A theory of planned behavior. En J. Kuhl y J. Beckmann (Eds). Action control: From cognition to behavior. Heilderberg: SpringerVerlag.

Ajzen, I. (1987). Attitudes, traits, and actions: Dispositional prediction of behavior. En L. Berkowitz (Ed.). Advances in experimental social psychology, 20. New York: Academic Press.

Ajzen, I. (1988) Attitudes, personality and behavior. Bristol: Open University Press.

Ajzen, I. y Fishbein, M (1980). Understanding attitudes and predicting behavior. New York: Prentice Hall. 
Arribas, S.; Arruza, J.A.; González, O.; Telletxea, S. Validación de una escala reducida de utilidad percibida de la práctica de la actividad física y el deporte. Revista Internacional de Ciencias del Deporte. 7(3), 34-48 http://www.cafyd.com/REVISTA/00704.pdf

Ajzen, I. y Madden, T.J. (1986). Prediction of goal-directed behavior: the role of intention, percived control and prior behavior. Journal of Experimental Social Psychology, 26, 305328.

Arkin, H. y Colton, R. (1962). Tables for stadisticians. New York: Barnes \& Noble.

Arribas, S. (2004). La práctica de la Actividad Física y el Deporte (PAFYD) en escolares de 15-18 años de Gipuzkoa: Creencias sobre su utilidad y relación con la orientación motivacional, Diversión y Satisfacción. Bilbo: Ed. UPV/EHU.

Bandura, A. (1982). Self-efficacy mechanisms in human agency. American Psychologist, 37, 122-147.

Cronbach, L.J. (1951). Coefficient alpha and the internal structure of test. Psikometrika, 16, 297-334.

Eagly, H. y Chaikens, S. (1993). The Psychology of attitudes, Harcourt Bran Janovich, Orlando.

Eiser, J.R. (1988). Attribution theory and social cognition. En J. Jaspars et al (Eds.). Attribution theory and research: coneptual and social dimensions. London: Academic Press.

Fishbein, M. y Ajzen, I. (1975) Belief, attitudes, intention and behaviour. An introduction to theory and research. Addison-Wesley, Massachusset.

García Cueto, E., Gallo, P. y Miranda, R. (1998). Bondad de ajuste en el análisis factorial confirmatorio. Psicothema, 10, 717-724.

García, L.F., Ruiz, M.A., y Abad, F.J. (2003). Factor structure of the Spanish WAIS-III. Psicothema, 15, 155-160.

Gondar, J.E. (2002). Modelado de ecuaciones Estructurales. Madrid: DMI.

Kuhl, J. (1982). The expentancy value approach within the theory of social motivation: elaborations, extensions, critique. En N.T. Feather (Ed.). Expectations and actions. Hillsdale: Erlbaum.

Martínez, R. (1996). Psicometría. Teoría de los test psicológicos y educativos. Madrid: Síntesis.

Nunnally, J. C. (1976). Psychometric theory. New York: McGraw-Hill.

Sánchez Bañuelos, F. (1987). Estudio del diferencial semántico en la actitud hacia el ejercicio físico realizado en las clases de Educación Física y el de carácter voluntario, en una muestra de alumnos de 3으 de BUP. Revista de investigación y Documentación sobre las ciencias de la EF y del Deporte, 2, 122-138. 
Arribas, S.; Arruza, J.A.; González, O.; Telletxea, S. Validación de una escala reducida de utilidad percibida de la práctica de la actividad física y el deporte. Revista Internacional de Ciencias del Deporte. 7(3), 34-48 http://www.cafyd.com/REVISTA/00704.pdf

Sánchez Bañuelos, F., Mendizábal, S. y Velasco, M. (2000). Diseño y validación de una escala para la medición de la utilidad percibida de la práctica del deporte. Infocoes, vol. IV, 2, 3-23.

Sandín, B., Chorot, P., Santed, M.A. y Valiente, R.M. (2003). Análisis factorial confirmatorio del Indice de Sensibilidad a la Ansiedad para niños. Psicothema,14, 333-339.

Vallerand, R.J. y Ricer, F. (1988). On the use of the Causal Dimension Scale in a field Setting: A test with confirmatory factor analysis in success and failure situations. Journal of Personality and Social Psychology, 54, 704-712. 
Arribas, S.; Arruza, J.A.; González, O.; Telletxea, S. Validación de una escala reducida de utilidad percibida de la práctica de la actividad física y el deporte. Revista Internacional de Ciencias del Deporte. 7(3), 34-48 http://www.cafyd.com/REVISTA/00704.pdf

\section{Anexo 1}

Cuestionario reducido sobre las creencias de utilidad de la práctica física y el deporte (CRUPPAFYD) (adaptada de Sánchez et al. 2000)

\section{La práctica del deporte sirve para:}

01- Olvidar las preocupaciones.

0-10-20-30-40-50-60-70-80-90-100

02- Desarrollar un mejor concepto de uno/a mismo/a.

0-10-20-30-40-50-60-70-80-90-100

03- Mejorar o mantener la forma física.

0-10-20-30-40-50-60-70-80-90-100

04- Lograr una mayor seguridad en uno/a mismo/a.

0-10-20-30-40-50-60-70-80-90-100

05- Desarrollar el afán de superación.

0-10-20-30-40-50-60-70-80-90-100

06- Comunicarse con los/as demás.

0-10-20-30-40-50-60-70-80-90-100

07- Ayudar mejor a los/as demás.

0-10-20-30-40-50-60-70-80-90-100

08- Desahogarse.

0-10-20-30-40-50-60-70-80-90-100

09- Dar salida al afán competitivo.

0-10-20-30-40-50-60-70-80-90-100

10- Hacer nuevos/as amigos/as.

0-10-20-30-40-50-60-70-80-90-100

11- Expresarse con libertad.

0-10-20-30-40-50-60-70-80-90-100

12- Mejorar y mantener la buena salud.

0-10-20-30-40-50-60-70-80-90-100 